\title{
Role of head-mounted displays in enhancing vestibular rehabilitation effects: Comment on "Evaluation of the effectiveness of a Virtual Reality-based exercise program for Unilateral Peripheral Vestibular Deficit"
}

\author{
Alessandro Micarelli ${ }^{\mathrm{a}, \mathrm{b}}$, Andrea Viziano ${ }^{\mathrm{a}, *}$ and Marco Alessandrini ${ }^{\mathrm{a}}$ \\ a Department of Clinical Sciences and Translational Medicine, 'Tor Vergata' University, Rome, Italy \\ ${ }^{\mathrm{b}}$ ITER Center for Balance and Rehabilitation Research (ICBRR); Rome, Italy
}

\section{Dear Editor,}

We read with great interest the work by Rosiak et al. "Evaluation of the effectiveness of a Virtual Realitybased exercise program for Unilateral Peripheral Vestibular Deficit" [6]. In this work, the effectiveness of a hybrid, virtual reality (VR)-based vestibular rehabilitation program was assessed in patients with peripheral vestibular dysfunction and compared to visual feedback using static posturography. Patients undergoing VR-based training showed more favorable outcomes with regard to self-reported outcomes, while both groups demonstrated improvements in posturographic parameters.

This study adds valuable knowledge to the expanding field of VR implementation in vestibular rehabilitation. Such an approach has become popular, due to promising results in transferring cognitive and motor skills acquired in virtual environments, such

\footnotetext{
*Corresponding author: Dr. Andrea Viziano, Department of Clinical Sciences and Translational Medicine, University of Rome 'Tor Vergata', Italy, Via Montpellier, 1; E sud Tower - 00133 Rome - Italy. Tel.: +0039 06 20903472; Fax: +0039 0620902930 ; E-mail: andrea.viziano@gmail.com.
}

as games or challenging visual scenarios, to reallife situations, while keeping patients engaged and motivated [4].

In the field of VR-based vestibular rehabilitation, recent evidence advocated for the use of headmounted displays (HMD) in a home-based gaming program coupled with conventional adaptation, habituation and substitution exercises [5]. Unilateral vestibular hypofunction patients demonstrated a significant improvement in posturography parameters and self-report measures such as the Dizziness Handicap Inventory; interestingly, also VOR gain was significantly increased in patients undergoing VRbased gameplay, with respect to their peers treated with conventional rehabilitation alone [5]. The use of HMD-based exercises has the advantage of delivering images as the highest possible resolution, while following the user's movements [3]. Visual information can be altered, asking patients to perform tasks in visually enriched environments, and vestibular stimulation can be added by incorporating head movements [1], facilitating central pre-programmed eye movements during active tasks which may contribute to gaze stability [5]. The enhancing effects of 
HMD on VOR improvement are in line with previous experiences, highlighting changes in dynamic visual acuity to reflect efficient compensatory mechanisms after vestibular impairment [2]. Exercises requiring eye and head movement while fixating on a visual target may induce VOR adaptation and/or facilitation of other types of eye movements improving gaze stability, posing a challenge (sensory mismatch) to the central nervous system, which then attempts to resolve the challenge [2,3].

The use of HMD has drawn some criticism due to movement restrictions, limited visual field and the possible onset of nausea and sickness [3]. However, those limitations in VR technology were assessed in the clinical field by means of the Simulator Sickness Questionnaire and did not represent an obstacle in completing the rehabilitation program, with significant reduction in sickness scores along the treatment, reflecting habituation to VR stimuli, which seems to occur regardless of the technique used $[1,5,6]$.

Less is known about possible variations in longterm rehabilitation outcomes; to this regard, it is of relevance that patients who were treated with HMDbased exercises maintained improvements in VOR gain and self-report measures as far as 12 months after discontinuation [7]. Such experiences seem to confirm that patient-tailored, VR-based vestibular rehabilitation may induce long-lasting adaptations in the central nervous system and that patients can remain free of symptoms after long periods of time. We believe that, due to the possibility of task-oriented learning in a comfortable environment, HMD-based exercise programs should be taken into consideration, compared to more time-consuming and expensive VR-based techniques, as a useful addition to conventional rehabilitation exercises.

\section{References}

[1] K.A. Alahmari, P.J. Sparto, G.F. Marchetti, M.S. Redfern, J.M. Furman and S.L. Whitney, Comparison of virtual reality based therapy with customized vestibular physical therapy for the treatment of vestibular disorders, IEEE Trans Neural Syst Rehabil Eng 22 (2014), 389-399.

[2] R.A. Clendaniel, The effects of habituation and gaze stability exercises in the treatment of unilateral vestibular hypofunction: A preliminary results, J Neurol Phys Ther 34 (2010), 111-116.

[3] V. Gatica-Rojas and G. Mendez-Rebolledo, Virtual reality interface devices in the reorganization of neural networks in the brain of patients with neurological diseases, Neural Regen $\operatorname{Res} 9$ (2014), 888-896.

[4] E.A. Keshner and J. Fung, The quest to apply VR technology to rehabilitation: Tribulations and treasures, J Vestib Res $\mathbf{2 7}$ (2017), 1-5.

[5] A. Micarelli, A. Viziano, I. Augimeri, D. Micarelli and M. Alessandrini, Three-dimensional head-mounted gaming task procedure maximizes effects of vestibular rehabilitation in unilateral vestibular hypofunction: A randomized controlled pilot trial, Int J Rehabil Res 40 (2017), 325-332.

[6] O. Rosiak, K. Krajewski, M. Woszczak and M. JozefowiczKorczynska, Evaluation of the effectiveness of a Virtual Reality-based exercise program for Unilateral Peripheral Vestibular Deficit, J Vestib Res (2019) Jan 28. doi: 10.3233/ VES-180647

[7] A. Viziano, A. Micarelli, I. Augimeri, D. Micarelli and M. Alessandrini, Long-term effects of vestibular rehabilitation and head-mounted gaming task procedure in unilateral vestibular hypofunction: A 12-month follow-up of a randomized controlled trial, Clin Rehabil 33 (2019), 24-33.

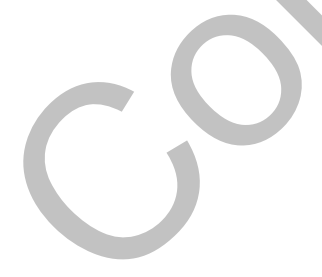

